\section{TREATMENT OF HEMOLYSIS WITH INTRAVENOUS UNFRACTIONATED HEPARIN USING AN ANTIFACTOR Xa-BASED MONITORING PROTOCOL \\ To the Editor:}

Management of hemolysis after implantation of continuous-flow left ventricular assist devices is associated with high mortality rates. Available treatment agents, including thrombolytics, glycoprotein IIb/IIIa inhibitors, and intravenous unfractionated heparin (IV-UFH), have not been able to preclude the need for device exchange or urgent cardiac transplantation. ${ }^{1-3}$ For patients receiving IV-UFH therapy, monitoring with antifactor Xa (anti-FXa) rather than activated partial thromboplastin time (aPTT) may improve outcomes.

We recently used anti-FXa to monitor IV-UFH treatment in a 67-year-old man with ischemic cardiomyopathy and a HeartMate II implant (Thoratec Corporation, Pleasanton, Calif) as a bridge to transplantation with an Interagency Registry for Mechanically Assisted Circulatory Support profile 4. His course was uncomplicated until a Staphylococcus driveline infection developed 1 year following implant. Chronic suppressive antibiotic therapy was initiated. He was admitted to our hospital 1 month later with peak lactate dehydrogenase $2800 \mathrm{U} / \mathrm{L}$ (reference range, 250-750 U/L) and hemoglobinuria with plasma free hemoglobin $50 \mathrm{mg} / \mathrm{dL}$, despite international normalized ratio of 2.2 and $81 \mathrm{mg}$ aspirin. No device alarms or evidence of end-organ dysfunction or hemodynamic compromise were present. An echocardiography-based ramp test showed a normal left ventricular enddiastolic dimension/speed slope of $-0.32 \quad(-0.16$ or greater suggests obstruction to flow). Because he met criteria for major hemolysis without evidence of obstruction to flow, he was treated with IV-UFH. His baseline aPTT was 43 seconds (reference range,

Clotting Cascade

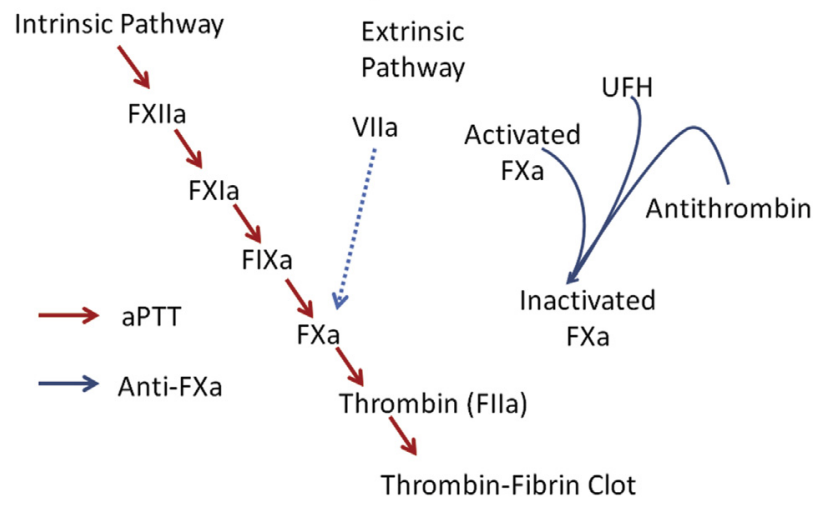

FIGURE 1. Antifactor Xa (Anti-FXa) determines anticoagulant activity by measuring the ability of the heparin-antithrombin complex to inhibit activated coagulation factor X. Measuring the inhibition of a single enzyme is a more direct measure of unfractionated heparin $(U F H)$ activity than activated partial thromboplastin time (aPTT), which assesses the intrinsic coagulation and final common pathway.

22-37 seconds). Our heparin infusion protocol monitors anti-FXa to achieve levels of 0.35 to $0.7 \mathrm{U} / \mathrm{mL}$. His average anti-FXa level was $0.42 \mathrm{U} / \mathrm{mL}$ despite corresponding aPTT levels $>240$ seconds. Under this protocol, hemoglobinuria resolved after 48 hours, and lactate dehydrogenase improved to 702 $\mathrm{U} / \mathrm{L}$, his baseline value. No evidence of recurrent hemolysis was observed through $>120$ days of follow-up. Anticoagulation was intensified to a goal international normalized ratio of 2.5 to 3 with $325 \mathrm{mg}$ aspirin.

The high rate of discordance between aPTT and anti-FXa has been previously reported. ${ }^{4,5}$ Anti-FXa represents a more direct measure of UFH activity, because it measures the inhibition of a single enzyme; that is, the ability of heparin-antithrombin complex to inhibit activated coagulation factor $\mathrm{X},{ }^{4}$ whereas aPTT reflects multiple steps in the coagulation cascade (Figure 1). In particular, aPTT dose-response to IV-UFH can be affected by warfarin coadministration, lupus anticoagulant, liver disease, and various factor deficiencies. To investigate the discordance in our patient, we measured factors II, V, VII, VIII, IX, X, XI, and XII and found low factor XII (36\%; reference range, $50 \%-186 \%$ ). Patients with factor XII deficiency have marked prolongation in aPTT but do not exhibit a bleeding diathesis. Monitoring of anti-FXa levels is unaffected by low factor XII and thus provided a more accurate reflection of intensity of anticoagulation in our patient.

To our knowledge, this is the first report of successful medical management of continuous-flow left ventricular assist device-related hemolysis based on anti-FXa monitoring. This case highlights the potential for successful medical management in selected patients, particularly when substantial risk factors for surgical device exchange are present.

Sirtaz Adatya, $M D$

Ranjit John, MD

Peter M. Eckman, MD

Cardiology Division

Department of Medicine

University of Minnesota

Minneapolis, Minn

\section{References}

1. Schlendorf K, Patel CB, Gehrig T, Kiefer TL, Felker GM, Hernandez AF, et al. Thrombolytic therapy for thrombosis of continuous flow ventricular assist devices. J Card Fail. 2014; 20:91-7.

2. Sylvia L, Ordway L, Pham DT, DeNofrio D Kiernan M. Bivalirudin for treatment of LVAD thrombosis: a case series. ASAIO J. 2014; 60:744-7.

3. Starling RC, Moazami N, Silvestry SC, Ewald G, Rogers JG, Milano CA, et al. Unexpected abrupt 
increase in left ventricular assist device thrombosis. N Engl J Med. 2014;370:33-40.

4. Mcllvennan CK, Page RL II, Ambardekar AV Brieke A, Lindenfeld J. Activated partial thromboplastin time overestimates anti-coagulation in left ventricular assist device patients. J Heart Lung Transplant. 2014;33:1312-4.

5. Adatya S, Uriel N, Yarmohammadi H, Holley CT, Feng A, Roy SS, et al. Anti-factor Xa and activated partial thromboplastin time measurements for heparin monitoring in mechanical circulatory support. J Am Coll Cardiol: Heart Fail. March 3, 2015 [Epub ahead of print].

\section{http://dx.doi.org/10.1016/ j.jtcvs.2015.02.029}

\section{FIBRINOGEN CONCENTRATE AS FIRST-LINE THERAPY IN CHILDREN UNDERGOING CARDIAC SURGERY: PROMISING PERSPECTIVES}

\section{To the Editor:}

Several questions remain unresolved regarding fibrinogen supplementation in bleeding situations, especially in children undergoing cardiac surgery. These questions include the component that should be used for fibrinogen supplementation; the safe and effective fibrinogen level to be targeted; and the test that should be used to guide fibrinogen administration.

In a recent issue of the Journal, Galas and colleagues ${ }^{1}$ first compared fibrinogen concentrate with cryoprecipitate as a first-line therapy for postbypass bleeding children. Although their study was not sufficiently powered to detect any significant difference in the incidence of postoperative bleeding and blood product transfusion requirements, relevant information should be highlighted from their study.

First, cryoprecipitate is no longer available in most European countries; thus, fresh frozen plasma (FFP) is still usually used as the first step in the transfusion algorithms. Despite the huge variability reported among cryoprecipitate units (3.0 to $9.0 \mathrm{~g} / \mathrm{L}){ }^{2}$ this variability within the infraphysiologic ranges is also observed among FFP bags $(0.9$ to $3.2 \mathrm{~g} / \mathrm{L}){ }^{3}$ which would explain why $\geq 20$ to $30 \mathrm{~mL} / \mathrm{kg}$ of
FFP should be administered in bleeding patients to significantly increase the fibrinogen levels. The results that would have been obtained in a similar study comparing FFP with fibrinogen concentrate would probably have reported a significant decrease in blood loss and blood transfusion requirements in children treated with fibrinogen concentrate.

Second, about $40 \%$ of children included in both groups received additional doses of cryoprecipitate, which could argue that the initial dose of both cryoprecipitate $(10 \mathrm{~mL} / \mathrm{kg})$ and fibrinogen concentrate $(60 \mathrm{mg} / \mathrm{kg})$ was not adequate to significantly increase the fibrinogen concentration within the first hour after administration. In addition, these results could be explained by the relatively low fibrinogen trigger used by the investigators $(<1.0 \mathrm{~g} / \mathrm{L})$ and the increased fibrinogen consumption observed in bleeding situations. Although no dose-response relationship has been studied, Rahe-Meyer and colleagues ${ }^{4}$ observed that fibrinogen concentrate, used at a dose of 90 to $100 \mathrm{mg} / \mathrm{kg}$, significantly decreased blood loss and the transfusion requirements in adults undergoing major aortic replacement surgery. In a recent animal trauma model, Martini and colleagues ${ }^{5}$ confirmed that $>100 \mathrm{mg} / \mathrm{kg}$ fibrinogen concentrate should be used to reach

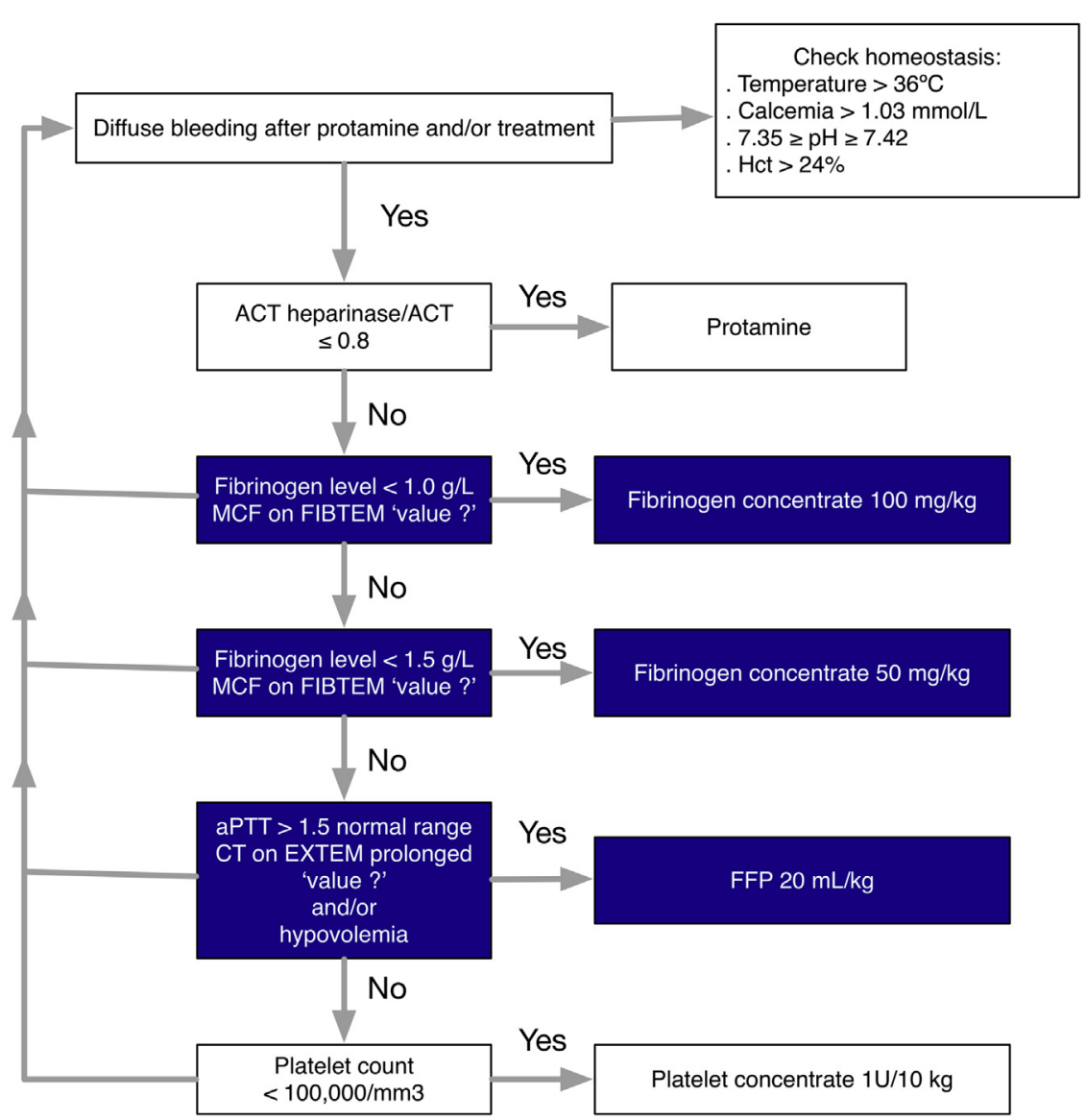

FIGURE 1. Proposed algorithm for the management of postbypass bleeding in children undergoing cardiac surgery. Blue boxes correspond to propositions that should be confirmed in future studies. Hct, Hematocrit; ACT, activated clotting time; $M C F$, maximal clot firmness; FIBTEM, a ROTEM assay (Tem International); $a P T T$, activated partial thromboplastin time; $C T$, clotting time; EXTEM, a ROTEM assay (Tem International); FFP, fresh frozen plasma. 